\title{
Static postural control assessment during pregnancy
}

\author{
ANA S. MOCCELLIN¹ | FERNANDA G.S.A. NORA² | PAULA H.L. COSTA | PATRICIA DRIUSSO³ | \\ 1 Universidade Federal de Sergipe, Lagarto, SE, Brazil. \\ 2 Universidade Federal de Goiás, Goiânia, GO, Brazil. \\ 3 Universidade Federal de São Carlos, São Carlos, SP, Brazil.
}

Correspondence to: Ana S. Moccellin, Universidade Federal de Sergipe, Rua Padre Álvares Pitangueira, 248, Centro, Lagarto, SE, BRAZIL. email: anamoccellin@ufs.br

\section{AT A GLANCE}

This study advances the current literature by investigating changes in postural control during pregnancy using a larger number of dependent variables associated with balance.

PUBLICATION DATA

Received 04 Sep 2014

Accepted 21 Feb 2015

Published 23 Feb 2015
BACKGROUND: The hormonal and anatomic changes during pregnancy affect the musculoskeletal system and may lead to instability of static postural control and increased risk of falls.

AIM: The aim of this study was to analyze changes in static postural control during the three trimesters of pregnancy, using variables derived from the center of pressure.

METHOD: This is a descriptive study in which posturographic tests were applied in four still standing conditions, for three trials, with a combination of different visual conditions (eyes open/eyes closed) and support base configurations on 20 non-pregnant women $(\mathrm{C})$ and 13 pregnant women during the gestational period (G1, G2 and G3). For static postural control assessment, a force plate (Bertec®) was used, and the variables analyzed were center of pressure area, displacement amplitude, displacement velocity and sway frequency.

RESULTS: A decrease in medial-lateral center of pressure sway frequency in the tandem with the right leg in front condition from the first to the third trimester was identified. There was also a positive significant correlation between mass and anterior-posterior sway frequency in the tandem with the right leg in front condition during the first trimester. A positive significant correlation between mass and anterior-posterior sway frequency was also identified in double-leg with eyes closed condition during the second trimester. No other significant effects were identified.

CONCLUSION: The results can suggest that some postural conditions, like double-leg stance with eyes closed and double-leg stance with feet aligned in tandem condition, can interfere on postural stability, mainly due to mass gain during pregnancy.

\section{INTRODUCTION}

During pregnancy, the female body undergoes many hormonal and anatomic changes, which may cause musculoskeletal complaints, in addition to affecting the pregnant woman's balance (Heckman \& Sassard, 1994; Ireland \& Ott, 2000; Vullo et al.,1996).

The increased mobility of the sacroiliac joint and pubic symphysis caused mainly by increased concentrations of the hormone relaxin between the $10^{\text {th }}$ and the $12^{\text {th }}$ week of pregnancy, along with the normal weight gain that occurs during pregnancy, may cause discomfort in joints of the pelvis, hip, knees and feet (Alvarez et al.,1988; Marnach et al.,2003; Paul et al., 1996; Ritchie, 2003). Approximately half of the weight gain is concentrated in the abdominal area anterior to the line of gravity, shifting the center of gravity (CG), which may contribute to postural instability (Butler et al., 2006; Niska et al., 1997). 
The impact of pregnancy on the musculoskeletal system results in adjustments of the static posture of women, which may impair their everyday tasks and increase the risk of falls (Dunning et al., 2003; Jang et al., 2008). However, few studies (Butler et al., 2006; Oliveira et al., 2009; Ribas \& Guirro, 2007) evaluated changes in postural control during pregnancy and they differ with respect to the control pattern adopted, interfering in a preventive intervention.

Oliveira et al. (2009) analyzed changes in body sway over the course of pregnancy by stabilogram and observed a decrease in postural control in situations of a reduced support base or with eyes closed. Ribas and Guirro (2007) analyzed plantar pressure and postural balance during the three trimesters of pregnancy and found a significant reduction on postural balance in the third trimester, associated with greater anterior-posterior displacement during this period. Butler et al. (2006) concluded that postural stability declines gradually during pregnancy and remains diminished at 6 to 8 weeks after delivery. This study also indicated that there is an increased dependency on visual cues to maintain balance during pregnancy.

Many of these studies used few center of pressure (COP) variables to explain postural control in pregnant women, thus, the purpose of the present study was to analyze changes in static postural control during the three trimesters, using variables derived from the COP, as well as observing relationships between these variables and anthropometric characteristics.

\section{METHODS}

\section{Participants}

Participants were recruited through local radio, television and newspaper announcements. To be included in the study, participants had to be nonsmokers, nondrinkers, not have undergone any surgeries on the spine, pelvis, hip or knee and had no previous conditions that could affect postural stability. In addition, participants should present low-risk and a single-fetus pregnancy, with no incidents during the gestational period.

Thirteen women were selected and completed the study $\left(M_{a g e}=26.07\right.$ years; age range $=5.64$ years). To establish a control baseline 20 non-pregnant women were recruited, constituting the control group ( $M_{\text {age }}=26.07$ years; age range $=3.98$ years). The study was approved by the Ethics Committee on Human Research at the Federal University of São Carlos (report \#280/2009) as determined by the resolution 196/96 of the National Health Council.

\section{Experimental Procedure}

After providing informed consent, participants answered a questionnaire to record personal data, lifestyle, personal history, medications, gynecological and obstetrical history. Then, anthopometric and static posturographic measurements were collected.

Women in the control group $(\mathrm{C})$ were measured only once, and these measures were compared to $\mathrm{G} 1$, since during the first trimester there is little influence of hormones and weight gain (Marnach et al., 2003; Ritchie, 2003). Pregnant women were evaluated at three different times, between the $10^{\text {th }}-14^{\text {th }}(\mathrm{G} 1), 22^{\text {nd }-24^{\text {th }}}(\mathrm{G} 2)$ and $32^{\text {nd }}-34^{\text {th }}(G 3)$ gestational weeks, according to last menstrual period (Alexander et al., 1990) and/or the 
first ultrasound during pregnancy (Rossavick \& Fishburne, 1989). Volunteers were barefoot during the evaluations. Evaluations of $\mathrm{C}, \mathrm{G} 1, \mathrm{G} 2$ and $\mathrm{G} 3$ followed exactly the same protocol.

\section{Apparatus}

For the static posturographic assessment measurements a force plate (Bertec Corporation) was used. The force plate was calibrated for horizontal anterior-posterior (AP), horizontal medial-lateral (ML) and vertical directions prior to data collection.

\section{Conditions}

The static posturographic assessment occurred under 4 conditions: double-leg stance with feet together and eyes opened (DEO) and closed (DEC), and double-leg stance with feet aligned in tandem with eyes open, alternating right leg (TRL) and left leg (TLL) in the front. The TRL and TLL conditions were used to assess postural control in situations with reduction of medial-lateral basis. Participants were instructed to stare at a fixed target at eye-level located two meters away from the force plate during assessment. Three 60-s trials for each condition were performed. The sampling rate of the COP signal was set at $100 \mathrm{~Hz}$. For this study the posturography standardization procedure recommended by Duarte and Freitas (2010) was used. Throughout the evaluation, a physical therapist remained next to the volunteer for instruction and safety.

\section{Dependent variables}

Anthropometric measures. Height (meters), body mass (kilograms) and pregnant body mass index (BMI) were collected. The pregnant BMI was calculated based on Atalah's table (Atalah et al., 1997), that takes into account the initial nutritional status of pregnant women (underweight, appropriate, overweight or obesity) and weight range recommended by each trimester.

Static posturographic measures. Variables analyzed were COP area (analytically evaluated by an ellipse containing $85.35 \%$ of the sample data), COP displacement (AP and $\mathrm{ML}$ ), COP velocity (AP and $\mathrm{ML}$ ) and COP sway frequency (AP and $\mathrm{ML}$ ). The COP parameters were processed using the MatLab software (MATH WORKS version 7.6.0.324, R2008a). Data were filtered with a 1 st order Butterworth, low-pass filter, cutoff frequency of $5 \mathrm{~Hz}$. The scores in each of the three trials were averaged and used in the statistcal analyses.

\section{Data Analysis}

Data were analyzed using the Statistica software. Nonparametric tests were used, since Shapiro-Wilk tests indicated variables did not demonstrate normal distribution. Friedman tests were used to determine changes within pregnant women over time $(\mathrm{G} 1, \mathrm{G} 2$, $\mathrm{G} 3$ ), and on positive cases Wilcoxon tests was used to identify differences. Differences between pregnant (G1) and control participants (C) were investigated with Mann-Whitney tests. The relationship between body mass and static posturographic measures was investigated using the Spearman tests. The level of significance was set to $5 \%(p \leq 0.05)$. Data are expressed as mean \pm standard deviation. 


\section{RESULTS}

\section{Anthropometric measures}

Table 1 presents age, height, body mass, and BMl of women in the control group $(C)$ and pregnant women during $\mathrm{G} 1, \mathrm{G} 2$, and $\mathrm{G} 3$. There was significant difference in body mass between $\mathrm{C}$ and $\mathrm{G} 1(p=.04), \mathrm{G} 1$ and $\mathrm{G} 3(p<.01), \mathrm{G} 2$ and $\mathrm{G} 3(p<.01)$. There was also significant differences in BMl between all trimesters $(p<.05)$. The mean weight gain from first to third trimester was $10.49( \pm 1.59) \mathrm{kg}$. The pregnant women maintained a BMI within limits considered appropriate throughout gestational weeks (Atalah et al., 1997).

Table 1 - Characteristics of pregnant and control subject groups

\begin{tabular}{|c|c|c|c|c|}
\hline Characteristics & Control & $1^{\text {st }}$ trimester & $2^{\text {nd }}$ trimester & $3^{\text {rd }}$ trimester \\
\hline Gestational week & - & $13.08 \pm 2.14$ & $23.23 \pm 1.59$ & $33.38 \pm 1.19$ \\
\hline Age (years) & $26.07 \pm 3.98$ & $29.15 \pm 5.64$ & $29.23 \pm 5.79$ & $29.46 \pm 5.83$ \\
\hline Height (m) & $1.63 \pm 0.07$ & $1.64 \pm 0.09$ & $1.64 \pm 0.09$ & $1.64 \pm 0.09$ \\
\hline Body mass $(\mathrm{kg})$ & $59.21 \pm 10.75$ & $66.24 \pm 13.34^{\star \mathrm{C}}$ & $71.26 \pm 13.49$ & $76.73 \pm 14.93^{\star^{1,2}}$ \\
\hline BMI $\left(\mathrm{kg} / \mathrm{m}^{2}\right)$ & $22.25 \pm 3.60$ & $24.60 \pm 4.39^{* \mathrm{C}}$ & $26.52 \pm 4.33^{* 1}$ & $28.47 \pm 4.66^{\star 1,2}$ \\
\hline
\end{tabular}

\section{Static posturographic measures}

Figure 1 represents the mean values of the COP areas in the four conditions analyzed. There were no significant differences between $C$ and $G 1$, or between $G 1, G 2$ and $\mathrm{G} 3$.

There were no significant differences in COP displacement AP/ML or in COP velocity ML/AP in any of the four conditions between $C$ and $\mathrm{G} 1$, or between the trimesters. COP sway frequency ML/AP in each of the four conditions is shown in Figure 2 . The only significant difference identified was in COP sway frequency ML between G1 and G3 in the TRL condition ( $p=.014$ ) (Fig. 2 A). On the same figure, the sway frequency reference values for adults are shown (Soames \& Atha, 1982). In DEO and DEC conditions, the ML and AP sway frequencies were close to minimum reference values. However, in TRL and TLL conditions, $C$ and $\mathrm{G} 1$ were close to the maximum reference values for $\mathrm{ML}$, while in terms of AP sway, averages of both groups lie well within the reference values. 
Brazilian Journal of Motor Behavior

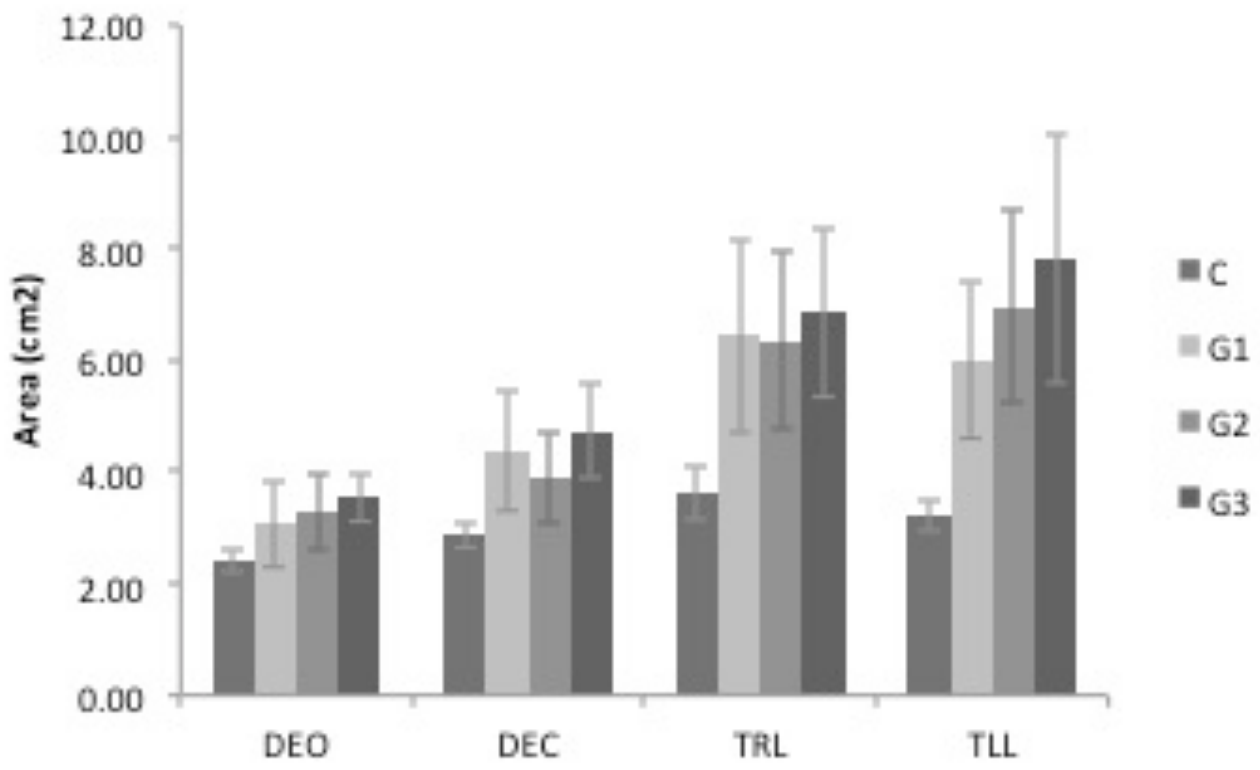

Figure 1. COP areas $\left(\mathrm{cm}^{2}\right)$ for control group (C), first (G1), second (G2) and third (G3) trimesters. DEO: double-leg eyes open; DEC: double-leg eyes closed; TRL: tandem right leg; TLL: tandem left leg.

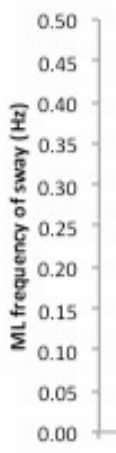

DEO

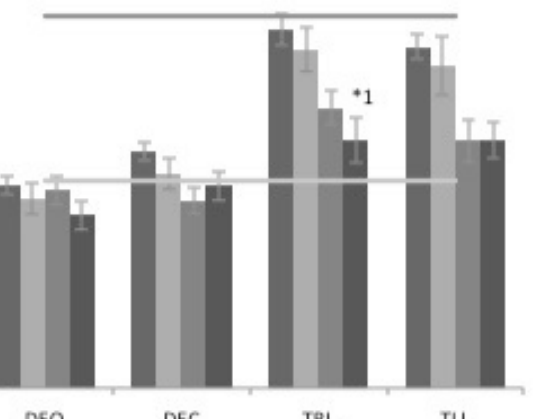

TRL

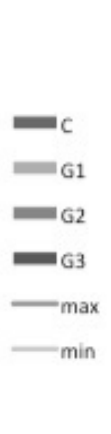

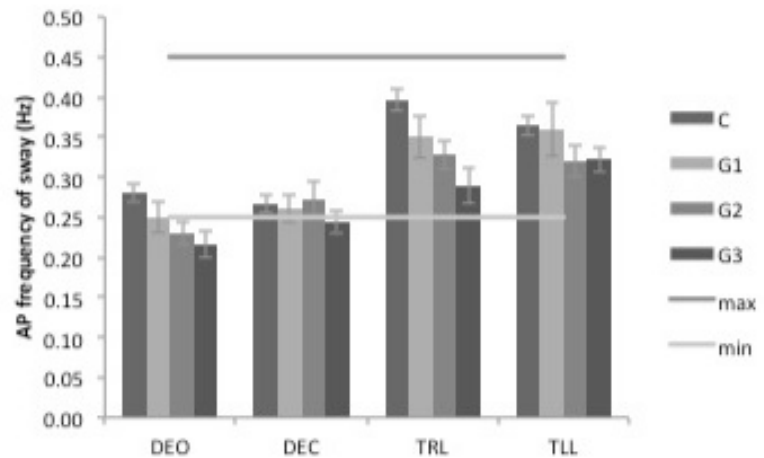

TRL

Figure 2. COP sway frequencies $(\mathrm{Hz})$ for control group (C), first (G1), second (G2) and third (G3) trimesters. A: medial-lateral (ML) sway frequency; B: anterior-posterior (AP) sway frequency. DEO: double-leg eyes open; DEC: double-leg eyes closed; TRL: tandem right leg; TLL: tandem left leg. Max: maximum reference value in adults $(0.45 \mathrm{~Hz})$, Min: minimum reference value in adults $(0.25 \mathrm{~Hz})$, according to Soames \& Atha22. * Significant Friedman test $(\mathrm{p}<0.05)$.

There was positive significant correlation $(p<0.05)$ between mass and AP sway frequency in the TRL condition for $\mathrm{G} 1(r=0.681)$ and in $\mathrm{BEC}$ condition for $\mathrm{G} 2(r=0.680)$. 


\section{DISCUSSION}

Postural balance assessment through different protocols (support base configurations and visual condition) may contribute to the development of therapeutic methods to prevent postural instability and falls during pregnancy. In the present study, we assessed anthropometric and static posturographic changes throughout the gestational period.

In terms of anthropometric measures, we identified differences in body mass between the control group and women in the first trimester of pregnancy. Additionally, body mass for women in the third trimester differed from body mass for women in the first and second trimester. These findings indicate a progressive increase in body mass, as expected. A similar pattern was observed for pregnant BMI. We identified differences in BMI between the control group and women in the first trimester of pregnancy. Additionally, BMI increased gradually through the trimesters. Also, as expected. The enlarging uterus, fetus and breasts, the increased blood volume, extravasation of extracellular fluid and water retention are the main causes of weight gain during pregnancy (Ireland \& Ott, 2000). However, $20 \%$ of this weight gain might increase joint overload by as much as $100 \%$ (Ritchie, 2003). On average, a woman will gain approximately $11 \mathrm{~kg}$ during pregnancy, but only $30-40 \%$ of pregnant women gain weight within the ideal recommended range (To \& Cheung, 1998). In this study, women had a mass gain within the recommended range $(10.49 \pm 1.59 \mathrm{~kg})$.

With respect to static posturographic measures, we identified a decrease in medial-lateral center of pressure sway frequency in the tandem with the right leg in front condition from the first to the third trimester indicating a increase in stability. A positive significant correlation between mass and anterior-posterior sway frequency in the tandem with the right leg in front condition during the first trimester. A positive significant correlation between mass and anterior-posterior sway frequency was also identified in double-leg with eyes closed condition during the second trimester. No other significant effects were identified.

It was expected that women in the first trimester would have a greater center of pressure (COP) area when compared with women in the control group because ligamentous laxity in sacroiliac joint and pubic symphysis increased on the first trimester, in order to favor the passage of the fetus during labor. This laxity may also occur in feet joints, causing postural instability. The relaxin hormone has been identified, in some studies, as a contributor to these changes (Laros, 1991; MacLennan, 1991; Ritchie, 2003). Its concentration is elevated during the first trimester and then decline early in the second trimester to a level that remains stable throughout the rest of the pregnancy and into labor (Heckman \& Sassard, 1994). Other authors also found a correlation between ligamentous laxity and the estrogen hormone (Charlton et al., 2001).

Our results show similarity between anterior-posterior (AP) and medial-lateral (ML) COP displacement in double-leg stance, which can be explained by the fact that, when feet are kept together, the support base is analogously configured as a square with the center of gravity (CG) representing the midpoint between the two directions. Likewise, it is believed that by separating feet, the support base expands, which decreases the ML displacement in relation to AP (Mochizuki et al., 2006). Ribas and Guirro (2007) analyzed the plantar pressure and postural balance during pregnancy, in double-leg stance with feet 
apart and open eyes. It was observed that the AP displacement amplitudes in the third trimester were greater compared to the first trimester, whereas in the ML direction there was no significant difference between trimesters. Mochizuki et al. (2006) investigated, in healthy adults, changes in postural sway (ML and AP displacement) associated with changes in the support base dimensions. The volunteers had higher AP displacement values when compared to $\mathrm{ML}$ displacement when placed in a larger support area. However, by reducing the area to a square, both the ML and AP direction values increased, which was also observed in our study.

During pregnancy, velocities in DEO and DEC conditions remained constant in both $\mathrm{ML}$ and $\mathrm{AP}$ directions. If we observe the $\mathrm{ML}$ and $\mathrm{AP}$ displacement in these same conditions, we will see that they were similar in both the DEO and DEC as G1 to G3. This was because the COP displacement velocity in those conditions was maintained or slightly increased. If we compare the velocities of double-leg feet together and double-leg feet in line (tandem) conditions, we can conclude that velocities of adjustments to maintain postural equilibrium with lower support base conditions are necessarily higher.

Besides COP variability and velocity, the sway frequency was also used to examine the postural control system. Regarding the AP and ML frequency of COP sway, lower frequency values indicate lower difference between the COM and COP, therefore, reflect a greater postural stability (Duarte \& Freitas, 2010). The results show that control group did not present different frequencies of COP sway compared to the values found in the first trimester. This is interesting, since it was expected that in control group the sway frequencies would be lower due to greater postural stability found in this group. Thus, the behavior of postural control cannot be described taking into account only isolated COP variables. The reference values for the frequencies of COP sway presented are predominantly found in healthy adults (Soames \& Atha, 1982). That does not mean that it is inappropriate to have values below the minimum. However, values above the maximum could imply relative postural control instability.

Our results were not consistent with recent studies cited to evaluate the static postural control in pregnant women. The differences found may be due to use of different assessment protocols and lack of standardization in methods of COP analysis, such as number of trials, testing time, distance from the visual field and sampling rates, or because the small sample size due to the high sample loss. It is suggested that further studies use larger sample sizes and assess postural control of pregnant women in the presence of a slight balance perturbation, which could mimic real life situations.

In conclusion, our findings can suggest that some postural conditions, like doubleleg stance with eyes closed and double-leg support with feet aligned in tandem condition, can interfere with postural stability, potentially due to increases in body mass during pregnancy. The clinical application of our results suggest pregnant women should practice exercises using different support base configurations and to encourage the use of a slightly enlarged support base (increasing the ML and AP support base), ensuring an adequate postural control during pregnancy. 


\section{REFERENCES}

1. Alvarez, R., Stokes, I.A.F., Asprinio, D.E., Trevino, S., \& Braun, T. (1988). Dimensional changes of the feet in pregnancy. The Journal of Bone \& Joint Surgery, 70(2), 271-4.

2. Atalah, S.E., Castillo, L.C., Castro, S.R., \& Aldea, P.A. (1997). Propuesta de un nuevo estándar de evaluación nutricional en embarazadas. Revista Médica de Chile, 125, 142936.

3. Butler, E.E., Colón, I., Druzin, M.L., \& Rose, J. (2006). Postural equilibrium during pregnancy: decreased stability with an increased reliance on visual cues. American Journal of Obstetrics \& Gynecology, 195(4):1104-8.

4. Charlton, W.P.H., Coslett-Charlton, L.M., \& Ciccotti, M.G. (2001). Correlation of estradiol in pregnancy and anterior cruciate ligament laxity. Clinical Orthopaedics and Related Research, 1(387):165-170.

5. Duarte, M., \& Freitas, S.M.S.F. (2010). Revisão sobre posturografia baseada em plataforma de força para avaliação do equilíbrio. Revista Brasileira de Fisioterapia, 14(3):183-92.

6. Dunning, K., LeMasters, G., Levin, L., Bhattacharya, A., Alterman, T., \& Lordo, K. (2003). Falls in workers during pregnancy: risk factors, job hazards, and high risk occupations. American Journal of Industrial Medicine, 44(6):664-72.

7. Heckman, J.D., \& Sassard, R. (1994). Musculoskeletal considerations in pregnancy. The Journal of Bone \& Joint Surgery, 76(11):1720-30.

8. Ireland, M.L., \& Ott, S.M. (2000). The effects of pregnancy on the musculoskeletal system. Clinical Orthopaedics and Related Research, 372:169-79.

9. Jang, J., Hsiao, K.T., \& Hsiao-Wecksler, E.T. (2008). Balance (perceived and actual) and preferred stance width during pregnancy. Clinical Biomechanics, 23(4):468-76.

10. Laros, R.K.Jr. (1991). Physiology of normal pregnancy. In: Wilson JR, Carrington ER. Obstetrics \& Gynecology, Ed 9. St. Louis: Mosby Year Book; 242p.

11. MacLennan, A.H. (1991). The role of the hormone relaxin in human reproduction and pelvic girdle relaxation. Scandinavian Journal of Rheumatology, Suppl. 88:7-15.

12. Marnach, M.L., Ramin, K.D., Ramsey, P.S., Song, S.W., Stensland, J.J., \& An, K.N. (2003). Characterization of the relationship between joint laxity and maternal hormones in pregnancy. Obstetrics \& Gynecology, 101(2):331-5.

13. Mochizuki, L., Duarte, M., Amadio, A.C., Zatsiorsky, V.M., \& Latash, M.L. (2006). Changes in postural sway and its fractions in conditions of postural instability. Journal of Applied Biomechanics, 22:51-60.

14. Niska, M., Sofer, D., Porat, A., Howard, C.B., Levi, A., \& Meizner, I. (1997). Planter foot pressures in pregnant women. Israel Journal of Medical Sciences, 33(2):139-46.

15. Oliveira, L.F., Vieira, T.M.M., Macedo, A.R., Simpson, D.M., \& Nadal, J. (2009). Postural sway changes during pregnancy: a descriptive study using stabilometry. European Journal of Obstetrics \& Gynecology and Reproductive Biology, 147:25-8. 
Brazilian Journal of Motor Behavior

16. Paul, J.A., Sallé, H., \& Frings-Dresen, M.H.W. (1996). Effect of posture on hip joint moment during pregnancy, while performing a standing task. Clinical Biomechanics, 11(2):111-5.

17. Ribas, S.I., \& Guirro, E.C.O. (2007). Análise da pressão plantar e do equilíbrio postural em diferentes fases da gestação. Revista Brasileira de Fisioterapia, 11(5):391-6.

18. Ritchie, J.R. (2003). Orthopedic considerations during pregnancy. Clinical Obstetrics \& Gynecology, 46(2):456-66.

19. Rossavick, L.K., \& Fishburne, J.I. (1989). Conceptional age, menstrual age, and ultrasound age: A second trimester comparison of pregnancies of known conceptional date with pregnancies dated from the last menstrual period. Obstetrics \& Gynecology, 73:243-9.

20. Soames, R.W., \& Atha, J. (1982). The spectral characteristics of postural sway behavior. European Journal of Applied Physiology, 49:169-77.

21. To, W.W.K., \& Cheung, W. (1998). The relationship between weight gain in pregnancy, birth-weight and postpartum weight retention. Journal of Obstetrics \& Gynaecology Research, 38(2):176-9.

22. Vullo, V.J., Richardson, J.K., \& Hurvitz, E.A. (1996). Hip, knee, and foot pain during pregnancy and the postpartum period. The Journal of Family Practice, 43(1):63-8.

Citation: Moccellin AS, Nora FGSA, Costa PHL, Driusso P. Static postural control assessment during pregnancy. BJMB. 2015: 9(1): 1-9.

Editor: Joao A. C. Barros, California State University Fullerton, Fullerton, CA, USA.

Copyright: (C) 2015 Moccellin, Nora, Costa and Driusso and BJMB. This is an open-access article distributed under the terms of the Creative Commons Attribution-NonCommercial-NoDerivatives 4.0 International License which permits unrestricted use, distribution, and reproduction in any medium, provided the original author and source are credited. Funding: This project was supported by Conselho Nacional de Desenvolvimento Científico e Tecnológico (CNPq, process \#130391/2010-7) awarded to Ana Silvia Moccellin.

Competing interests: The authors have declared that no competing interests exist.

Download: http://socibracom.com/bjmb/index.php/bjmb/issue/view/10 